\title{
ON LIE GROUPS AS QUASI-KÄHLER MANIFOLDS WITH KILLING NORDEN METRIC
}

\author{
MANCHO MANEV, DIMITAR MEKEROV
}

\begin{abstract}
A 6-parametric family of 6-dimensional quasi-Kähler manifolds with Norden metric is constructed on a Lie group. This family is characterized geometrically.
\end{abstract}

\section{Contents}

Introduction

1. Almost Complex Manifolds with Norden Metric

1.1. Preliminaries

1.2. Curvature properties

1.3. Isotropic Kähler manifolds

2. Lie groups as Quasi-Kähler manifolds with

Killing Norden metric

3. The Lie Group as a 6-Dimensional $\mathcal{W}_{3}$-Manifold

4. Geometric characteristics of the constructed manifold 7

4.1. The components of the tensor $F \quad 7$

4.2. The square norm of the Nijenhuis tensor 8

4.3. The square norm of $\nabla J \quad 8$

4.4. The components of $R \quad 8$

4.5. The components of $\rho$ and the value of $\tau \quad 9$

4.6. The sectional curvatures and the holomorphic bisectional curvature $\quad 10$

4.7. The isotropic-Kählerian property 11

References

\section{INTRODUCTION}

It is a fundamental fact that on an almost complex manifold with Hermitian metric (almost Hermitian manifold), the action of the almost complex structure on the tangent space at each point of the manifold is isometry. There is another kind of metric, called a Norden metric or a $B$-metric on an almost complex manifold, such that the action of the almost complex structure is anti-isometry with respect to the metric. Such a manifold is called an almost complex manifold with Norden

Date: $2006 / 11 / 11$.

2000 Mathematics Subject Classification. 53C15, 53C50 (Primary); 32Q60, 53C55 (Secondary).

Key words and phrases. almost complex manifold, Norden metric, quasi-Kähler manifold, indefinite metric, non-integrable almost complex structure, Lie group. 
metric [1] or with $B$-metric [2]. See also [6] for generalized $B$-manifolds. It is known [1] that these manifolds are classified into eight classes.

The purpose of the present paper is to exhibit, by construction, almost complex structures with Norden metric on Lie groups as 6-manifolds, which are of a certain class, called the class of the quasi-Kähler manifolds with Norden metrics. This 6 -parametric family of manifolds is characterized geometrically.

The case of the lower dimension 4 is considered in [5] and [9].

\section{Almost Complex Manifolds with Norden Metric}

1.1. Preliminaries. Let $(M, J, g)$ be a $2 n$-dimensional almost complex manifold with Norden metric, i. e. $J$ is an almost complex structure and $g$ is a metric on $M$ such that

$$
J^{2} X=-X, \quad g(J X, J Y)=-g(X, Y)
$$

for all differentiable vector fields $X, Y$ on $M$, i. e. $X, Y \in \mathfrak{X}(M)$.

The associated metric $\tilde{g}$ of $g$ on $M$ given by $\tilde{g}(X, Y)=g(X, J Y)$ for all $X, Y \in$ $\mathfrak{X}(M)$ is a Norden metric, too. Both metrics are necessarily of signature $(n, n)$. The manifold $(M, J, \tilde{g})$ is an almost complex manifold with Norden metric, too.

Further, $X, Y, Z, U(x, y, z, u$, respectively) will stand for arbitrary differentiable vector fields on $M$ (vectors in $T_{p} M, p \in M$, respectively).

The Levi-Civita connection of $g$ is denoted by $\nabla$. The tensor filed $F$ of type $(0,3)$ on $M$ is defined by

$$
F(X, Y, Z)=g\left(\left(\nabla_{X} J\right) Y, Z\right) .
$$

It has the following symmetries

$$
F(X, Y, Z)=F(X, Z, Y)=F(X, J Y, J Z) .
$$

Further, let $\left\{e_{i}\right\}(i=1,2, \ldots, 2 n)$ be an arbitrary basis of $T_{p} M$ at a point $p$ of $M$. The components of the inverse matrix of $g$ are denoted by $g^{i j}$ with respect to the basis $\left\{e_{i}\right\}$.

The Lie form $\theta$ associated with $F$ is defined by

$$
\theta(z)=g^{i j} F\left(e_{i}, e_{j}, z\right) .
$$

A classification of the considered manifolds with respect to $F$ is given in [1]. Eight classes of almost complex manifolds with Norden metric are characterized there according to the properties of $F$. The three basic classes are given as follows

$$
\begin{aligned}
& \mathcal{W}_{1}: F(x, y, z)=\frac{1}{4 n}\{g(x, y) \theta(z)+g(x, z) \theta(y) \\
& +g(x, J y) \theta(J z)+g(x, J z) \theta(J y)\} ; \\
& \begin{aligned}
\mathcal{W}_{2}: & \underset{x, y, z}{\mathfrak{S}} F(x, y, J z)=0, \quad \theta=0 ; \\
\mathcal{W}_{3}: & \underset{x, y, z}{\mathfrak{S}} F(x, y, z)=0,
\end{aligned}
\end{aligned}
$$

where $\mathfrak{S}$ is the cyclic sum by three arguments.

The special class $\mathcal{W}_{0}$ of the Kähler manifolds with Norden metric belonging to any other class is determined by the condition $F=0$. 
ON LIE GROUPS AS QUASI-KÄHLER MANIFOLDS WITH KILLING NORDEN METRIC 3

1.2. Curvature properties. Let $R$ be the curvature tensor field of $\nabla$ defined by

$$
R(X, Y) Z=\nabla_{X} \nabla_{Y} Z-\nabla_{Y} \nabla_{X} Z-\nabla_{[X, Y]} Z .
$$

The corresponding tensor field of type $(0,4)$ is determined as follows

$$
R(X, Y, Z, U)=g(R(X, Y) Z, U) .
$$

The Ricci tensor $\rho$ and the scalar curvature $\tau$ are defined as usual by

$$
\rho(y, z)=g^{i j} R\left(e_{i}, y, z, e_{j}\right), \quad \tau=g^{i j} \rho\left(e_{i}, e_{j}\right) .
$$

Let $\alpha=\{x, y\}$ be a non-degenerate 2-plane (i. e. $\pi_{1}(x, y, y, x)=g(x, x) g(y, y)-$ $\left.g(x, y)^{2} \neq 0\right)$ spanned by vectors $x, y \in T_{p} M, p \in M$. Then, it is known, the sectional curvature of $\alpha$ is defined by the following equation

$$
k(\alpha)=k(x, y)=\frac{R(x, y, y, x)}{\pi_{1}(x, y, y, x)} .
$$

The basic sectional curvatures in $T_{p} M$ with an almost complex structure and a Norden metric $g$ are

- holomorphic sectional curvatures if $J \alpha=\alpha$;

- totally real sectional curvatures if $J \alpha \perp \alpha$ with respect to $g$.

In [4], a holomorphic bisectional curvature $h(x, y)$ for a pair of holomorphic 2planes $\alpha_{1}=\{x, J x\}$ and $\alpha_{2}=\{y, J y\}$ is defined by

$$
h(x, y)=-\frac{R(x, J x, y, J y)}{\sqrt{\pi_{1}(x, J x, x, J x) \pi_{1}(y, J y, y, J y)}},
$$

where $x, y$ do not lie along the totally isotropic directions, i. e. both of the couples $(g(x, x), g(x, J x))$ and $(g(y, y), g(y, J y))$ are different from the couple $(0,0)$. The holomorphic bisectional curvature is invariant with respect to the basis of the 2planes $\alpha_{1}$ and $\alpha_{2}$. In particular, if $\alpha_{1}=\alpha_{2}$, then the holomorphic bisectional curvature coincides with the holomorphic sectional curvature of the 2-plane $\alpha_{1}=$ $\alpha_{2}$.

1.3. Isotropic Kähler manifolds. The square norm $\|\nabla J\|^{2}$ of $\nabla J$ is defined in [3] by

$$
\|\nabla J\|^{2}=g^{i j} g^{k l} g\left(\left(\nabla_{e_{i}} J\right) e_{k},\left(\nabla_{e_{j}} J\right) e_{l}\right) .
$$

Having in mind the definition (1.2) of the tensor $F$ and the properties (1.3), we obtain the following equation for the square norm of $\nabla J$

$$
\|\nabla J\|^{2}=g^{i j} g^{k l} g^{p q} F_{i k p} F_{j l q},
$$

where $F_{i k p}=F\left(e_{i}, e_{k}, e_{p}\right)$.

Definition $1.1([8])$. An almost complex manifold with Norden metric satisfying the condition $\|\nabla J\|^{2}=0$ is called an isotropic Kähler manifold with Norden metric.

Remark 1.1. It is clear, if a manifold belongs to the class $\mathcal{W}_{0}$, then it is isotropic Kählerian but the inverse statement is not always true. 


\section{LIE GROUPS AS QUASI-KÄHLER MANIFOLDS WITH} KiLLing NoRDEN METRIC

The only class of the three basic classes, where the almost complex structure is not integrable, is the class $\mathcal{W}_{3}$ - the class of the quasi-Kähler manifolds with Norden metric.

Let us remark that the definitional condition from (1.5) implies the vanishing of the Lie form $\theta$ for the class $\mathcal{W}_{3}$.

Let $V$ be a $2 n$-dimensional vector space and consider the structure of the Lie algebra defined by the brackets $\left[E_{i}, E_{j}\right]=C_{i j}^{k} E_{k}$, where $\left\{E_{1}, E_{2}, \ldots, E_{2 n}\right\}$ is a basis of $V$ and $C_{i j}^{k} \in \mathbb{R}$.

Let $G$ be the associated connected Lie group and $\left\{X_{1}, X_{2}, \ldots, X_{2 n}\right\}$ be a global basis of left invariant vector fields induced by the basis of $V$. Then the Jacobi identity has the form

$$
\underset{X_{i}, X_{j}, X_{k}}{\mathfrak{S}}\left[\left[X_{i}, X_{j}\right], X_{k}\right]=0
$$

Next we define an almost complex structure by the conditions

$$
J X_{i}=X_{n+i}, \quad J X_{n+i}=-X_{i}, \quad i \in\{1,2, \ldots, n\} .
$$

Let us consider the left invariant metric defined by the following way

$$
\begin{aligned}
& g\left(X_{i}, X_{i}\right)=-g\left(X_{n+i}, X_{n+i}\right)=1, \quad i \in\{1,2, \ldots, n\} \\
& g\left(X_{j}, X_{k}\right)=0, \quad j \neq k \in\{1,2, \ldots, 2 n\} .
\end{aligned}
$$

The introduced metric is a Norden metric because of (2.2).

In this way, the induced $2 n$-dimensional manifold $(G, J, g)$ is an almost complex manifold with Norden metric, in short almost Norden manifold.

The condition the Norden metric $g$ be a Killing metric of the Lie group $G$ with the corresponding Lie algebra $\mathfrak{g}$ is $g(\operatorname{ad} X(Y), Z)=-g(Y, \operatorname{ad} X(Z)$, where $X, Y, Z \in \mathfrak{g}$ and $\operatorname{ad} X(Y)=[X, Y]$. It is equivalent to the condition the metric $g$ to be an invariant metric, i. e.

$$
g([X, Y], Z)+g([X, Z], Y)=0 .
$$

Theorem 2.1. If $(G, J, g)$ is an almost Norden manifold with a Killing metric $g$, then it is:

(i) a $\mathcal{W}_{3}$-manifold;

(ii) a locally symmetric manifold.

Proof. (i) Let $\nabla$ be the Levi-Civita connection of $g$. Then the following well-known condition is valid

$$
\begin{aligned}
2 g\left(\nabla_{X} Y, Z\right)= & X g(Y, Z)+Y g(X, Z)-Z g(X, Y) \\
& +g([X, Y], Z)+g([Z, X], Y)+g([Z, Y], X) .
\end{aligned}
$$

The last equation and (2.4) imply

$$
\nabla_{X_{i}} X_{j}=\frac{1}{2}\left[X_{i}, X_{j}\right], \quad i, j \in\{1,2, \ldots, 2 n\} .
$$

Then we receive consecutively

$$
\left(\nabla_{X_{i}} J\right) X_{j}=\nabla_{X_{i}} J X_{j}-J \nabla_{X_{i}} X_{j}=\frac{1}{2}\left(\left[X_{i}, J X_{j}\right]-J\left[X_{i}, X_{j}\right]\right),
$$




$$
F\left(X_{i}, X_{j}, X_{k}\right)=\frac{1}{2}\left\{g\left(\left[X_{i}, J X_{j}\right], X_{k}\right)-g\left(\left[X_{i}, X_{j}\right], J X_{k}\right)\right\} .
$$

According to (2.4), the last equation implies $\mathfrak{S}_{X_{i}, X_{j}, X_{k}} F\left(X_{i}, X_{j}, X_{k}\right)=0$, i. e. the manifold belongs to the class $\mathcal{W}_{3}$.

(ii) In [5] is given the following form of the curvature tensor

$$
\left.R\left(X_{i}, X_{j}, X_{k}, X_{l}\right)=-\frac{1}{4} g\left(\left[\left[X_{i}, X_{j}\right], X_{k}\right], X_{l}\right]\right) .
$$

Using the condition (2.4) for a Killing metric, we obtain

$$
R\left(X_{i}, X_{j}, X_{k}, X_{l}\right)=-\frac{1}{4} g\left(\left[X_{i}, X_{j}\right],\left[X_{k}, X_{l}\right]\right) .
$$

According to the constancy of the component $R_{i j k s}$ and (2.6) and (2.8), we get the covariant derivative of the tensor $R$ of type $(0,4)$ as follows

$$
\begin{aligned}
\left(\nabla_{X_{i}} R\right) & \left(X_{j}, X_{k}, X_{l}, X_{m}\right)= \\
=\frac{1}{8}\{ & \left.g\left(\left[\left[X_{i}, X_{j}\right], X_{k}\right]-\left[\left[X_{i}, X_{k}\right], X_{j}\right],\left[X_{l}, X_{m}\right]\right]\right) \\
& \left.\left.+g\left(\left[\left[X_{i}, X_{l}\right], X_{m}\right]-\left[\left[X_{i}, X_{m}\right], X_{l}\right],\left[X_{j}, X_{k}\right]\right]\right)\right\} .
\end{aligned}
$$

We apply the the Jacobi identity (2.1) to the double commutators. Then the equation (2.9) gets the form

$$
\begin{aligned}
\left(\nabla_{X_{i}} R\right)\left(X_{j}, X_{k}, X_{l}, X_{m}\right)=-\frac{1}{8}\{ & g\left(\left[X_{i},\left[X_{j}, X_{k}\right]\right],\left[X_{l}, X_{m}\right]\right) \\
& \left.\left.+g\left(\left[X_{i},\left[X_{l}, X_{m}\right]\right],\left[X_{j}, X_{k}\right]\right)\right)\right\} .
\end{aligned}
$$

Since $g$ is a Killing metric, then applying (2.4) to (2.10) we obtain the identity $\nabla R=0$, i. e. the manifold is locally symmetric.

\section{The Lie Group as a 6 -Dimensional $\mathcal{W}_{3}$-Manifold}

Let $(G, J, g)$ be a 6 -dimensional almost Norden manifold with Killing metric $g$. Having in mind Theorem 2.1 we assert that $(G, J, g)$ is a $\mathcal{W}_{3}$-manifold. Let the commutators have the following decomposition

$$
\left[X_{i}, X_{j}\right]=\gamma_{i j}^{k} X_{k}, \quad \gamma_{i j}^{k} \in \mathbb{R}, \quad i, j, k \in\{1,2, \ldots, 6\} .
$$

According to the condition (2.4) for a Killing metric $g$, the equations (3.1) take the form given in Table 1.

Further we consider the special case when the condition

$$
g\left(\left[X_{i}, X_{j}\right],\left[X_{k}, X_{l}\right]\right)=0
$$

for all mutually different indices $i, j, k, l$ in $\{1,2, \ldots, 6\}$ holds.

According to the Jacobi identity (2.1) and the condition (3.2), Table 1 is transformed to Table 2.

The Lie groups $G$ thus obtained are of a family which is characterized by six real parameters $\lambda_{i}(i=1, \ldots, 6)$. Therefore, for the manifold $(G, J, g)$ constructed above, we establish the truthfulness of the following 
TABLE 1. The Lie brackets with 20 parameters.

\begin{tabular}{||l||c|c|c||c|c|c||}
\hline & $X_{1}$ & $X_{2}$ & $X_{3}$ & $X_{4}$ & $X_{5}$ & $X_{6}$ \\
\hline \hline$\left[X_{2}, X_{3}\right]$ & $\lambda_{10}$ & & & $\lambda_{7}$ & $\lambda_{1}$ & $\lambda_{2}$ \\
\hline$\left[X_{3}, X_{1}\right]$ & & $\lambda_{10}$ & & $\lambda_{4}$ & $\lambda_{8}$ & $\lambda_{3}$ \\
\hline$\left[X_{1}, X_{2}\right]$ & & & $\lambda_{10}$ & $\lambda_{5}$ & $\lambda_{6}$ & $\lambda_{9}$ \\
\hline \hline$\left[X_{5}, X_{6}\right]$ & $\lambda_{17}$ & $\lambda_{14}$ & $\lambda_{15}$ & $\lambda_{20}$ & & \\
\hline$\left[X_{6}, X_{4}\right]$ & $\lambda_{11}$ & $\lambda_{18}$ & $\lambda_{16}$ & & $\lambda_{20}$ & \\
\hline$\left[X_{4}, X_{5}\right]$ & $\lambda_{12}$ & $\lambda_{13}$ & $\lambda_{19}$ & & & $\lambda_{20}$ \\
\hline \hline$\left[X_{1}, X_{4}\right]$ & & $\lambda_{5}$ & $-\lambda_{4}$ & & $-\lambda_{12}$ & $\lambda_{11}$ \\
\hline$\left[X_{1}, X_{5}\right]$ & & $\lambda_{6}$ & $-\lambda_{8}$ & $\lambda_{12}$ & & $-\lambda_{17}$ \\
\hline$\left[X_{1}, X_{6}\right]$ & & $\lambda_{9}$ & $-\lambda_{3}$ & $-\lambda_{11}$ & $\lambda_{17}$ & \\
\hline \hline$\left[X_{2}, X_{4}\right]$ & $-\lambda_{5}$ & & $\lambda_{7}$ & & $-\lambda_{13}$ & $\lambda_{18}$ \\
\hline$\left[X_{2}, X_{5}\right]$ & $-\lambda_{6}$ & & $\lambda_{1}$ & $\lambda_{13}$ & & $-\lambda_{14}$ \\
\hline$\left[X_{2}, X_{6}\right]$ & $-\lambda_{9}$ & & $\lambda_{2}$ & $-\lambda_{18}$ & $\lambda_{14}$ & \\
\hline \hline$\left[X_{3}, X_{4}\right]$ & $\lambda_{4}$ & $-\lambda_{7}$ & & & $-\lambda_{19}$ & $\lambda_{16}$ \\
\hline$\left[X_{3}, X_{5}\right]$ & $\lambda_{8}$ & $-\lambda_{1}$ & & $\lambda_{19}$ & & $-\lambda_{15}$ \\
\hline$\left[X_{3}, X_{6}\right]$ & $\lambda_{3}$ & $-\lambda_{2}$ & & $-\lambda_{16}$ & $\lambda_{15}$ & \\
\hline
\end{tabular}

TABle 2. The Lie brackets with 6 parameters.

\begin{tabular}{||c||c|c|c||c|c|c||}
\hline & $X_{1}$ & $X_{2}$ & $X_{3}$ & $X_{4}$ & $X_{5}$ & $X_{6}$ \\
\hline \hline$\left[X_{2}, X_{3}\right]$ & & & & & $\lambda_{1}$ & $\lambda_{2}$ \\
\hline$\left[X_{3}, X_{1}\right]$ & & & & $\lambda_{4}$ & & $\lambda_{3}$ \\
\hline$\left[X_{1}, X_{2}\right]$ & & & & $\lambda_{5}$ & $\lambda_{6}$ & \\
\hline \hline$\left[X_{5}, X_{6}\right]$ & & $-\lambda_{4}$ & $-\lambda_{5}$ & & & \\
\hline$\left[X_{6}, X_{4}\right]$ & $-\lambda_{1}$ & & $-\lambda_{6}$ & & & \\
\hline$\left[X_{4}, X_{5}\right]$ & $-\lambda_{2}$ & $-\lambda_{3}$ & & & & \\
\hline \hline$\left[X_{1}, X_{4}\right]$ & & $\lambda_{5}$ & $-\lambda_{4}$ & & $\lambda_{2}$ & $-\lambda_{1}$ \\
\hline$\left[X_{1}, X_{5}\right]$ & & $\lambda_{6}$ & & $-\lambda_{2}$ & & \\
\hline$\left[X_{1}, X_{6}\right]$ & & & $-\lambda_{3}$ & $\lambda_{1}$ & & \\
\hline \hline$\left[X_{2}, X_{4}\right]$ & $-\lambda_{5}$ & & & & $\lambda_{3}$ & \\
\hline$\left[X_{2}, X_{5}\right]$ & $-\lambda_{6}$ & & $\lambda_{1}$ & $-\lambda_{3}$ & & $\lambda_{4}$ \\
\hline$\left[X_{2}, X_{6}\right]$ & & & $\lambda_{2}$ & & $-\lambda_{4}$ & \\
\hline \hline$\left[X_{3}, X_{4}\right]$ & $\lambda_{4}$ & & & & & $-\lambda_{6}$ \\
\hline$\left[X_{3}, X_{5}\right]$ & & $-\lambda_{1}$ & & & & $\lambda_{5}$ \\
\hline$\left[X_{3}, X_{6}\right]$ & $\lambda_{3}$ & $-\lambda_{2}$ & & $\lambda_{6}$ & $-\lambda_{5}$ & \\
\hline
\end{tabular}

Theorem 3.1. Let $(G, J, g)$ be a 6-dimensional almost Norden manifold, where $G$ is a connected Lie group with corresponding Lie algebra $\mathfrak{g}$ determined by the global basis of left invariant vector fields $\left\{X_{1}, X_{2}, \ldots, X_{6}\right\} ; J$ is an almost complex structure defined by (2.2) and $g$ is an invariant Norden metric determined by (2.3) and (2.4). Then $(G, J, g)$ is a quasi-Kähler manifold with Norden metric if and only if $G$ belongs to the 6-parametric family of Lie groups determined by Table 2. 
For the constructed manifold the Killing form $B(X, Y)=\operatorname{tr}(\operatorname{ad} X$, ad $Y)$ has the following determinant

$$
\operatorname{det} B=64\left[\left(\lambda_{1}^{2}+\lambda_{2}^{2}+\lambda_{3}^{2}-\lambda_{4}^{2}-\lambda_{5}^{2}-\lambda_{6}^{2}\right)^{2}-4\left(\lambda_{1}^{2}-\lambda_{4}^{2}\right)\left(\lambda_{3}^{2}-\lambda_{6}^{2}\right)\right]^{3} .
$$

A special case when the holomorphic sectional curvatures are zero is considered in [7]. In the cited case we have $\lambda_{1}=\lambda_{4}, \lambda_{2}=\lambda_{5}, \lambda_{3}=\lambda_{6}$ and then the Killing form is degenerate.

Now, in the general case the question whether the Killing form $B$ can be a Norden metric is reasonable. The answer is negative because the setting of the condition $B$ to be a Norden metric implies $\lambda_{i}=0(i=1,2, \ldots, 6)$.

\section{Geometric Characteristics of the Constructed manifold}

Let $(G, J, g)$ be the 6 -dimensional $\mathcal{W}_{3}$-manifold introduced in the previous section.

4.1. The components of the tensor $F$. Then by direct calculations, having in mind (1.2), (2.2), (2.3), (2.4), (2.6), (2.7) and Table 2, we obtain the nonzero components of the tensor $F$ as follows

$$
\begin{aligned}
\lambda_{1} & =2 F_{116}=2 F_{161}=-2 F_{134}=-2 F_{143}=2 F_{223}=2 F_{232}=2 F_{256} \\
& =2 F_{265}=-F_{322}=-F_{355}=2 F_{413}=2 F_{431}=2 F_{446}=2 F_{464} \\
& =-2 F_{526}=-2 F_{562}=2 F_{535}=2 F_{553}=-F_{611}=-F_{644}, \\
\lambda_{2} & =-2 F_{115}=-2 F_{151}=2 F_{124}=2 F_{142}=F_{233}=F_{266}=-2 F_{323} \\
& =-2 F_{332}=-2 F_{356}=-2 F_{365}=-2 F_{412}=-2 F_{421}=-2 F_{445} \\
& =-2 F_{454}=F_{511}=F_{544}=-2 F_{626}=-2 F_{662}=2 F_{635}=2 F_{653}, \\
\lambda_{3} & =-F_{133}=-F_{166}=-2 F_{215}=-2 F_{251}=2 F_{224}=2 F_{242}=2 F_{313} \\
& =2 F_{331}=2 F_{346}=2 F_{364}=-F_{422}=-F_{455}=2 F_{512}=2 F_{521} \\
& =2 F_{545}=2 F_{554}=2 F_{616}=2 F_{661}=-2 F_{634}=-2 F_{643}, \\
\lambda_{4} & =-2 F_{113}=-2 F_{131}=-2 F_{146}=-2 F_{164}=-2 F_{226}=-2 F_{262} \\
& =2 F_{235}=2 F_{253}=F_{311}=F_{344}=2 F_{416}=2 F_{461}=-2 F_{434} \\
& =-2 F_{523}=-2 F_{443}=-2 F_{532}=-2 F_{556}=-2 F_{565}=F_{622}=F_{655}, \\
\lambda_{5} & =2 F_{112}=2 F_{121}=2 F_{145}=2 F_{154}=-F_{211}=-F_{244}=-2 F_{326} \\
& =-2 F_{362}=2 F_{335}=2 F_{353}=-2 F_{415}=-2 F_{451}=2 F_{424}=2 F_{442} \\
& =-F_{533}=-F_{566}=2 F_{623}=2 F_{632}=2 F_{656}=2 F_{665}, \\
\lambda_{6} & =F_{122}=F_{155}=-2 F_{212}=-2 F_{221}=-2 F_{245}=-2 F_{254}=2 F_{316} \\
& =2 F_{361}=-2 F_{334}=-2 F_{343}=F_{433}=F_{466}=-2 F_{515}=-2 F_{551} \\
& =2 F_{524}=2 F_{542}=-2 F_{613}=-2 F_{631}=-2 F_{646}=-2 F_{664} . \\
& =1 \mathrm{~V}
\end{aligned}
$$

where $F_{i j k}=F\left(X_{i}, X_{j}, X_{k}\right)$. 
4.2. The square norm of the Nijenhuis tensor. Let $N$ be the Nijenhuis tensor of the almost complex structure $J$ on $G$, i. e.

$$
N(X, Y)=[X, Y]+J[J X, Y]+J[X, J Y]-[J X, J Y], \quad X, Y \in \mathfrak{g} .
$$

Having in mind the equations in Table 2, we obtain the nonzero components $N_{i j}=$ $N\left(X_{i}, X_{j}\right)(i, j=1,2, \ldots, 6)$ as follows

$$
\begin{aligned}
& N_{23}=-N_{56}=2\left(\lambda_{4} X_{2}+\lambda_{5} X_{3}+\lambda_{1} X_{5}+\lambda_{2} X_{6}\right), \\
& N_{31}=-N_{64}=2\left(\lambda_{1} X_{1}+\lambda_{6} X_{3}+\lambda_{4} X_{4}+\lambda_{3} X_{6}\right), \\
& N_{12}=-N_{45}=2\left(\lambda_{2} X_{1}+\lambda_{3} X_{2}+\lambda_{5} X_{4}+\lambda_{6} X_{5}\right), \\
& N_{35}=-N_{26}=2\left(-\lambda_{1} X_{2}-\lambda_{2} X_{3}+\lambda_{4} X_{5}+\lambda_{5} X_{6}\right), \\
& N_{16}=-N_{34}=2\left(-\lambda_{4} X_{1}-\lambda_{3} X_{3}+\lambda_{1} X_{4}+\lambda_{6} X_{6}\right), \\
& N_{24}=-N_{15}=2\left(-\lambda_{5} X_{1}-\lambda_{6} X_{2}+\lambda_{2} X_{4}+\lambda_{3} X_{5}\right) .
\end{aligned}
$$

Therefore its square norm $\|N\|^{2}=g^{i k} g^{k s} g\left(N_{i j}, N_{k s}\right)$ vanishes, i. e. $\|N\|^{2}=0$, where the nonzero components of the inverse matrix of $g$ are the following

$$
g^{11}=g^{22}=g^{33}=-g^{44}=-g^{55}=-g^{66}=1 .
$$

Then we have the following

Proposition 4.1. The Nijenhuis tensor of the manifold $(G, J, g)$ is isotropic.

4.3. The square norm of $\nabla J$. According to (2.3), (4.1) and (4.4), from (1.12) we obtain that the square norm of $\nabla J$ is zero, i. e. $\|\nabla J\|^{2}=0$. Then we have the following

Proposition 4.2. The manifold $(G, J, g)$ is isotropic Kählerian.

4.4. The components of $R$. Let $R$ be the curvature tensor of type $(0,4)$ determined by (1.7) and (1.6) on $(G, J, g)$. We denote its components by $R_{i j k s}=$ $R\left(X_{i}, X_{j}, X_{k}, X_{s}\right) ; i, j, k, s \in\{1,2, \ldots, 6\}$. Using (2.6), (2.1), (2.8) and Table 2 we 
get the nonzero components of $R$ as follows

$$
\begin{aligned}
& R_{1441}=-\frac{1}{4}\left(\lambda_{1}^{2}+\lambda_{2}^{2}-\lambda_{4}^{2}-\lambda_{5}^{2}\right), \\
& R_{2552}=\frac{1}{4}\left(\lambda_{1}^{2}-\lambda_{3}^{2}-\lambda_{4}^{2}+\lambda_{6}^{2}\right), \\
& R_{3663}=\frac{1}{4}\left(\lambda_{2}^{2}+\lambda_{3}^{2}-\lambda_{5}^{2}-\lambda_{6}^{2}\right), \\
& R_{1221}=-\frac{1}{4}\left(\lambda_{5}^{2}+\lambda_{6}^{2}\right), \quad R_{1331}=-\frac{1}{4}\left(\lambda_{3}^{2}+\lambda_{4}^{2}\right), \\
& R_{1551}=-\frac{1}{4}\left(\lambda_{2}^{2}-\lambda_{6}^{2}\right), \quad R_{1661}=-\frac{1}{4}\left(\lambda_{1}^{2}-\lambda_{3}^{2}\right), \\
& R_{2332}=-\frac{1}{4}\left(\lambda_{1}^{2}+\lambda_{2}^{2}\right), \quad R_{2442}=-\frac{1}{4}\left(\lambda_{3}^{2}-\lambda_{5}^{2}\right), \\
& R_{2662}=\frac{1}{4}\left(\lambda_{2}^{2}-\lambda_{4}^{2}\right), \quad R_{3443}=\frac{1}{4}\left(\lambda_{4}^{2}-\lambda_{6}^{2}\right), \\
& R_{3553}=\frac{1}{4}\left(\lambda_{1}^{2}-\lambda_{5}^{2}\right), \quad R_{4554}=\frac{1}{4}\left(\lambda_{2}^{2}+\lambda_{3}^{2}\right), \\
& R_{4664}=\frac{1}{4}\left(\lambda_{1}^{2}+\lambda_{6}^{2}\right), \quad R_{5665}=\frac{1}{4}\left(\lambda_{4}^{2}+\lambda_{5}^{2}\right), \\
& R_{1561}=R_{2562}=R_{3563}=-R_{4564}=\frac{1}{4} \lambda_{1} \lambda_{2}, \\
& R_{1341}=R_{2342}=-R_{5345}=-R_{6346}=-\frac{1}{4} \lambda_{1} \lambda_{3}, \\
& R_{1361}=R_{2362}=-R_{4364}=-R_{5365}=\frac{1}{4} \lambda_{1} \lambda_{4}, \\
& R_{1261}=R_{3263}=-R_{4264}=-R_{5265}=-\frac{1}{4} \lambda_{1} \lambda_{5}, \\
& R_{2132}=-R_{4134}=-R_{5135}=-R_{6136}=\frac{1}{4} \lambda_{1} \lambda_{6}, \\
& R_{3123}=-R_{4124}=-R_{5125}=-R_{6126}=\frac{1}{4} \lambda_{2} \lambda_{3}, \\
& R_{1351}=R_{2352}=-R_{4354}=-R_{6356}=-\frac{1}{4} \lambda_{2} \lambda_{4}, \\
& R_{1251}=R_{3253}=-R_{4254}=-R_{6256}=\frac{1}{4} \lambda_{2} \lambda_{5}, \\
& R_{1241}=R_{3243}=-R_{5245}=-R_{6246}=-\frac{1}{4} \lambda_{2} \lambda_{6}, \\
& R_{1461}=R_{2462}=R_{3463}=-R_{5465}=\frac{1}{4} \lambda_{3} \lambda_{4}, \\
& R_{2152}=R_{3153}=-R_{4154}=-R_{6156}=-\frac{1}{4} \lambda_{3} \lambda_{5} \text {, } \\
& R_{2142}=R_{3143}=-R_{5145}=-R_{6146}=\frac{1}{4} \lambda_{3} \lambda_{6}, \\
& R_{1231}=-R_{4234}=-R_{5235}=-R_{6236}=\frac{1}{4} \lambda_{4} \lambda_{5} \text {, } \\
& R_{2162}=R_{3163}=-R_{4164}=-R_{5165}=-\frac{1}{4} \lambda_{4} \lambda_{6} \text {, } \\
& R_{1451}=R_{2452}=R_{3453}=-R_{6456}=\frac{1}{4} \lambda_{5} \lambda_{6} \text {. }
\end{aligned}
$$

4.5. The components of $\rho$ and the value of $\tau$. Having in mind (1.8), (4.4) and (4.5), we obtain the components $\rho_{i j}=\rho\left(X_{i}, X_{j}\right)(i, j=1,2, \ldots, 6)$ of the Ricci 
tensor $\rho$ and the the value of the scalar curvature $\tau$ as follows

$$
\begin{gathered}
\rho_{11}=\frac{1}{2}\left(\lambda_{1}^{2}+\lambda_{2}^{2}-\lambda_{3}^{2}-\lambda_{4}^{2}-\lambda_{5}^{2}-\lambda_{6}^{2}\right), \\
\rho_{22}=\frac{1}{2}\left(-\lambda_{1}^{2}-\lambda_{2}^{2}+\lambda_{3}^{2}+\lambda_{4}^{2}-\lambda_{5}^{2}-\lambda_{6}^{2}\right), \\
\rho_{33}=\frac{1}{2}\left(-\lambda_{1}^{2}-\lambda_{2}^{2}-\lambda_{3}^{2}-\lambda_{4}^{2}+\lambda_{5}^{2}+\lambda_{6}^{2}\right), \\
\rho_{44}=\frac{1}{2}\left(-\lambda_{1}^{2}-\lambda_{2}^{2}-\lambda_{3}^{2}+\lambda_{4}^{2}+\lambda_{5}^{2}-\lambda_{6}^{2}\right), \\
\rho_{55}=\frac{1}{2}\left(\lambda_{1}^{2}-\lambda_{2}^{2}-\lambda_{3}^{2}-\lambda_{4}^{2}-\lambda_{5}^{2}+\lambda_{6}^{2}\right), \\
\rho_{66}=\frac{1}{2}\left(-\lambda_{1}^{2}+\lambda_{2}^{2}+\lambda_{3}^{2}-\lambda_{4}^{2}-\lambda_{5}^{2}-\lambda_{6}^{2}\right), \\
\rho_{12}=\lambda_{2} \lambda_{3}, \quad \rho_{13}=\lambda_{1} \lambda_{6}, \quad \rho_{14}=\lambda_{3} \lambda_{6} ; \\
\rho_{15}=-\lambda_{3} \lambda_{5}, \quad \rho_{16}=-\lambda_{4} \lambda_{6}, \quad \rho_{23}=\lambda_{4} \lambda_{5} ; \\
\rho_{24}=-\lambda_{2} \lambda_{6}, \quad \rho_{25}=\lambda_{2} \lambda_{5}, \quad \rho_{26}=-\lambda_{1} \lambda_{5} ; \\
\rho_{34}=-\lambda_{1} \lambda_{3}, \quad \rho_{35}=-\lambda_{2} \lambda_{4}, \quad \rho_{36}=\lambda_{1} \lambda_{4} ; \\
\rho_{45}=\lambda_{5} \lambda_{6}, \quad \rho_{46}=\lambda_{3} \lambda_{4}, \quad \rho_{56}=\lambda_{1} \lambda_{2} ; \\
\quad \tau=0,
\end{gathered}
$$

i. e. the scalar curvature on $(G, J, g)$ is zero.

The last equation implies immediately

Proposition 4.3. The manifold $(G, J, g)$ is scalar flat.

4.6. The sectional curvatures and the holomorphic bisectional curvature. Let us consider the characteristic 2-planes $\alpha_{i j}$ spanned by the basis vectors $\left\{X_{i}, X_{j}\right\}$ at an arbitrary point of the manifold:

- holomorphic 2-planes: $\alpha_{14}, \alpha_{25}, \alpha_{36}$;

- pairs of totally real 2-planes: $\left(\alpha_{12}, \alpha_{45}\right) ;\left(\alpha_{13}, \alpha_{46}\right) ;\left(\alpha_{15}, \alpha_{24}\right) ;\left(\alpha_{16}, \alpha_{34}\right)$; $\left(\alpha_{23}, \alpha_{56}\right) ;\left(\alpha_{26}, \alpha_{35}\right)$.

Then, using (1.9), (2.3) and (4.5), we obtain the corresponding sectional curvatures

$$
\begin{array}{cl}
k\left(\alpha_{14}\right)=\frac{1}{4}\left(\lambda_{1}^{2}+\lambda_{2}^{2}-\lambda_{4}^{2}-\lambda_{5}^{2}\right), & k\left(\alpha_{25}\right)=-\frac{1}{4}\left(\lambda_{1}^{2}-\lambda_{3}^{2}-\lambda_{4}^{2}+\lambda_{6}^{2}\right), \\
k\left(\alpha_{36}\right)=-\frac{1}{4}\left(\lambda_{2}^{2}+\lambda_{3}^{2}-\lambda_{5}^{2}-\lambda_{6}^{2}\right) ; \\
k\left(\alpha_{12}\right)=-\frac{1}{4}\left(\lambda_{5}^{2}+\lambda_{6}^{2}\right), \quad k\left(\alpha_{45}\right)=\frac{1}{4}\left(\lambda_{2}^{2}+\lambda_{3}^{2}\right), \\
k\left(\alpha_{13}\right)=-\frac{1}{4}\left(\lambda_{3}^{2}+\lambda_{4}^{2}\right), & k\left(\alpha_{46}\right)=\frac{1}{4}\left(\lambda_{1}^{2}+\lambda_{6}^{2}\right), \\
k\left(\alpha_{15}\right)=\frac{1}{4}\left(\lambda_{2}^{2}-\lambda_{6}^{2}\right), & k\left(\alpha_{24}\right)=\frac{1}{4}\left(\lambda_{3}^{2}-\lambda_{5}^{2}\right), \\
k\left(\alpha_{16}\right)=\frac{1}{4}\left(\lambda_{1}^{2}-\lambda_{3}^{2}\right), & k\left(\alpha_{34}\right)=-\frac{1}{4}\left(\lambda_{4}^{2}-\lambda_{6}^{2}\right), \\
k\left(\alpha_{23}\right)=-\frac{1}{4}\left(\lambda_{1}^{2}+\lambda_{2}^{2}\right), & k\left(\alpha_{56}\right)=\frac{1}{4}\left(\lambda_{4}^{2}+\lambda_{5}^{2}\right), \\
k\left(\alpha_{26}\right)=-\frac{1}{4}\left(\lambda_{2}^{2}-\lambda_{4}^{2}\right), & k\left(\alpha_{35}\right)=-\frac{1}{4}\left(\lambda_{1}^{2}-\lambda_{5}^{2}\right) .
\end{array}
$$

Taking into account (1.10), (2.3) and (4.5), we obtain that the holomorphic bisectional curvature of the three pairs of the basis holomorphic 2-planes vanishes, i. e.

$$
h\left(\alpha_{14}, \alpha_{25}\right)=h\left(\alpha_{14}, \alpha_{36}\right)=h\left(\alpha_{25}, \alpha_{36}\right)=0 .
$$


ON LIE GROUPS AS QUASI-KÄHLER MANIFOLDS WITH KILLING NORDEN METRIC 11

4.7. The isotropic-Kählerian property. Having in mind Propositions 4.1-4.2 and Theorem 2.1, we give the following characteristics of the constructed manifold

Theorem 4.4. The manifold $(G, J, g)$ constructed as an $\mathcal{W}_{3}$-manifold with Killing metric in Theorem 3.1:

(i) is isotropic Kählerian;

(ii) has an isotropic Nijenhuis tensor;

(iii) is scalar flat;

(iv) is locally symmetric.

\section{REFERENCES}

[1] G. Ganchev and A. Borisov, Note on the Almost Complex Manifolds with a Norden Metric. Compt. Rend. Acad. Bulg. Sci. 39 (1986), no. 5, 31-34. 2

[2] G. Ganchev, K. Gribachev and V. Mihova, B-Connections and their Conformal Invariants on Conformally Kahler Manifolds with B-Metric. Publ. Inst. Math. (Beograd) (N.S.) 42 (1987), 107-121. 2

[3] E. García-Río and Y. Matsushita, Isotropic Kähler Structures on Engel 4-Manifolds. J. Geom. Phys. 33 (2000), 288-294. 3

[4] K. I. Gribachev, G. D. Djelepov and D. G. Mekerov, On Some Subclasses of Generalized B-Manifolds. Compt. Rend. Acad. Bulg. Sci. 38 (1985), no. 4, 437-440. 3

[5] K. Gribachev, M. Manev and D. Mekerov, A Lie group as a 4-dimensional quasi-Kahler manifold with Norden metric. JP J. Geom. Topol. 6 (2006), no. 1, 55-68. 2, 5

[6] K. I. Gribachev, D. G. Mekerov and G. D. Djelepov, Generalized B-Manifolds. Compt. Rend. Acad. Bulg. Sci. 38 (1985), no. 3, 299-302. 2

[7] M. Manev, K. Gribachev and D. Mekerov, On three-parametric Lie groups as quasi-Kähler manifolds with Killing Norden metric. In: Proc. of 8th International Workshop on Complex Structures and Vector Fields, Sofia, 2006 (to appear). 7

[8] D. Mekerov and M. Manev, On the geometry of quasi-Kähler manifolds with Norden metric. Nihonkai Math. J. 16 (2005), no. 2, 89-93. 3

[9] D. Mekerov, M. Manev and K. Gribachev, A Lie group as a 4-dimensional quasi-Kahler manifold with respect to a pair of Norden metrics. Results Math. 49 (2006), no. 1-2, 161-170. 2

University of Plovdiv, Faculty of Mathematics and Informatics, 236 Bulgaria Blvd., 4003 Plovdiv, Bulgaria, http://WWW. Fmi-Plovdiv. org/Manev

E-mail address: mmanev@uni-plovdiv.bg, mircho@uni-plovdiv.bg 